Quantum molecular dynamics simulations of uranium at high pressure and temperature

R. Q. Hood, L. H. Yang, J. A. Moriarty

February 11,2008

Physical Review B 
This document was prepared as an account of work sponsored by an agency of the United States government. Neither the United States government nor Lawrence Livermore National Security, LLC, nor any of their employees makes any warranty, expressed or implied, or assumes any legal liability or responsibility for the accuracy, completeness, or usefulness of any information, apparatus, product, or process disclosed, or represents that its use would not infringe privately owned rights. Reference herein to any specific commercial product, process, or service by trade name, trademark, manufacturer, or otherwise does not necessarily constitute or imply its endorsement, recommendation, or favoring by the United States government or Lawrence Livermore National Security, LLC. The views and opinions of authors expressed herein do not necessarily state or reflect those of the United States government or Lawrence Livermore National Security, LLC, and shall not be used for advertising or product endorsement purposes. 


\title{
Quantum molecular dynamics simulations of uranium at high pressure and temperature
}

\author{
Randolph Q. Hood, L. H. Yang, and John A. Moriarty \\ Lawrence Livermore National Laboratory, Livermore, CA 94550
}

\begin{abstract}
Constant-volume quantum molecular dynamics (QMD) simulations of uranium (U) have been carried out over a range of pressures and temperatures that span the experimentally observed solid orthorhombic $\alpha$-U, body-centered cubic (bcc), and liquid phases, using an ab initio plane-wave pseudopotential method within the generalized gradient approximation of density functional theory. A robust $\mathrm{U}$ pseudopotential has been constructed for these simulations that treats the 14 valence and outer-core electrons per atom necessary to calculate accurate structural and thermodynamic properties up to $100 \mathrm{GPa}$. Its validity has been checked by comparing low-temperature results with experimental data and all-electron full-potential linear-muffin-tin-orbital calculations of several different uranium solid structures. Calculated QMD energies and pressures for the equation of state of uranium in the solid and liquid phases are given, along with results for the Grüneisen parameter and the specific heat. We also present results for the radial distribution function, bondangle distribution function, electronic density of states, and liquid diffusion coefficient, as well as evidence for short-range order in the liquid.
\end{abstract}

PACS numbers: PACS: 71.15.Pd, 61.20.Ja, 71.20.Gj 


\section{INTRODUCTION}

Quantum molecular dynamics (QMD) simulation methods based upon density functional theory $^{1,2}$ (DFT) and the use of plane-wave ab initio pseudopotentials ${ }^{3-6}$ have been very successful in simulating the high-pressure and -temperature properties of low- $Z$ materials $(Z \leq 20)$, and more recently in several instances, higher- $Z$ materials $(21 \leq Z \leq 82)$ as well. In the case of metals, this includes $s p$-bonded simple metals such as aluminum ${ }^{7}$ and $\operatorname{tin}^{8}$ and $d$-bonded transition metals such as iron, ${ }^{9,10}$ molybdenum, ${ }^{11,12}$ and tantalum. ${ }^{13}$ In these materials one has mostly close-packed high-symmetry structures in the solid: facecentered cubic (fcc), body-centered cubic (bcc), and hexagonal close-packed (hcp). To the best of our knowledge, there are no published reports of QMD studies for more complex metals in which $f$ electrons participate in the bonding such as the early actinide elements, thorium through plutonium $(90 \leq Z \leq 94)$. These metals can be treated by DFT methods but are more challenging because they have narrow-band $5 f$ electrons with bandwidths of 1-3 eV, in comparison to transition metals with $d$ bandwidths of 3-10 eV and simple metals with even larger $s p$ bandwidths. These $5 f$ electrons have a dominate influence upon the electronic structure, imparting highly directional bonding and producing polymorphism and low-symmetry crystal structures not seen by other metals in the periodic table. ${ }^{14-16}$ In addition, any QMD simulation on an actinide metal is inherently more computationally demanding because of the number of electrons per atom that must be treated for accurate results: 12-16 (including the outer core $5 s$ and $5 p$ states), as compared to 1-4 for simple metals and 3-9 for transition metals. Since DFT-based QMD has been applied successfully in the latter cases, it is of great interest to know how this approach will fair when applied to the actinides. Uranium $(\mathrm{U})$ is a good starting point for such investigations. It has an interesting high-pressure phase diagram as well as many unusual properties near ambient pressure, and because $\mathrm{U}$ is both nontoxic and naturally occurring, there exists a great deal of experimental data with which simulations can be compared.

The narrow band itinerant $5 f$ electrons near the Fermi level in uranium give rise to several interesting and unique physical properties. These bands lead to a symmetry-breaking mechanism similar to a Peierls or Jahn-Teller distortion that lowers the total energy and results in an open, low-symmetry ground-state structure, denoted as $\alpha$-U, that is facecentered orthorhombic with two atoms per primitive cell. Below the temperature of $43 \mathrm{~K}$ 
uranium is unique among the elements in having a charge density wave (CDW) state s-19 $^{17-19}$ that is not induced by a spin-density wave, as is the case in chromium. ${ }^{20,21}$ Theoretically, the CDW in uranium has been attributed to the opening of gaps, ${ }^{22}$ a Peierls-like transition, at the Fermi level with its lattice distortion arising from a strong nesting of the fairly narrow $5 f$ bands. Recent theoretical calculations have predicted ${ }^{23}$ that the narrow $5 f$ electrons of solid uranium, which is non-magnetic in the bulk, give rise to a surface magnetic moment on the (001) surface. Rhodium is the only elemental solid where a surface magnetic moment has been observed experimentally in a solid that is nonmagnetic in the bulk. ${ }^{24,25}$ The electronphonon coupling in uranium is believed to be the source of additional unusual properties such as an intrinsically localized vibrational mode that was recently observed ${ }^{26}$ above 450 $\mathrm{K}$, and its rich superconducting features. ${ }^{27}$ Reviews of the physical properties of uranium have been given by Fisher ${ }^{28}$ and Lander et al. ${ }^{18}$

Regarding high-pressure properties, the phase diagram and equation of state (EOS) of uranium have been studied in piston-cylinder ${ }^{29}$ and diamond-anvil-cell ${ }^{30-33}$ (DAC) experiments to $100 \mathrm{GPa}$. The $\alpha$-U structure is observed to remain the stable phase at room temperature over this entire pressure range, ${ }^{32}$ while above $1050 \mathrm{~K}$ there is a prominent hightemperature bcc $(\gamma)$ phase $^{34}$ that has been observed in the DAC to $60 \mathrm{GPa} .{ }^{33}$ Below $4 \mathrm{GPa}$, there is also an intermediate tetragonal $(\beta)$ phase with a complex and still uncertain 30-atom per unit cell structure that exists over a small temperature range (940-1050 K at ambient pressure). ${ }^{35-37}$ An interesting feature of the bcc phase is that it is mechanically unstable at low temperature (with a negative $C^{\prime}$ shear modulus) over the whole 100 GPa pressure range, as DFT calculations have shown. ${ }^{16}$ Thus the bcc $\gamma$ phase is only stabilized at high temperature by thermal contributions, e.g., large anharmonic phonon effects and/or large electron-thermal effects. This situation is analogous to that in the Group-IVB transition metals titanium, zirconium, and hafnium, which all have high temperature bcc phases that are mechanically unstable at low temperature. Also, as in the Group-IVB metals, uranium melts out of the bcc structure up to high pressures. The melt curve in U has been measured in laser-heated DAC experiments to $100 \mathrm{GPa} .{ }^{31,33}$

Regarding corresponding high-pressure theory on uranium, there have been a number of DFT calculations of zero-temperature properties, as we shall discuss below. There has been comparatively little work, however, on the high-temperature phase diagram and EOS. One result of note was an early calculation of the melt curve ${ }^{31}$ using quantum-based effective 
pair potentials derived from model generalized pseudopotential theory ${ }^{38}$ (MGPT). While this treatment did lead to a quite reasonable melt curve, the potentials themselves suffer from two main shortcomings. First, they neglect non-isotropic, angular forces associated with the $5 f$-electron directional bonding. As a consequence, neither the $\alpha$-U ground state nor the mechanical instability of the bcc phase at low temperature are described. Second, no account of electron-thermal effects has been taken in either the potentials nor the melting calculation. Physically, one expects the high density of electronic states at the Fermi level arising from the narrow-band $5 f$ electrons to produce a strong coupling between the ion- and electron-thermal degrees of freedom for temperatures as low as melt, leading to temperaturedependent forces on the ions and an impact on the melt curve. Using DFT-based QMD we believe these shortcomings can be overcome. This approach treats the electrons and ions on a fundamental and more equal footing, so both the directional $5 f$-bonding and the temperature-dependent forces are rigorously treated. In our QMD simulations, the electrons and the ions are held in thermodynamic equilibrium, and the forces felt by the ions are calculated self-consistently on the fly by computing the eigenstates of the electronic Hamiltonian for each instantaneous position of the ions.

At the same time, a direct and accurate QMD simulation of the melt curve remains a formidable challenge for any high- $Z$ metal and to our knowledge none has yet been reported. In this regard, the melt-curve results published to date for simple and transition metals ${ }^{8-13}$ have all been indirectly determined, making extensive use of simple reference potentials and/or other approximate devices to obtain the result. In the present initial QMD study on uranium our focus will be on the high-temperature EOS in the relevant solid and liquid phases obtained using direct QMD simulations rather than on any such determination of the melt curve or other phase boundaries. Regarding such applications, we have also recently developed complementary multi-ion MGPT potentials for U, including three- and four-ion potentials but based on zero-temperature electrons, and we are working to develop a capability to obtain full temperature-dependent MGPT potentials using QMD simulations. ${ }^{39}$ This latter work will be reported separately at a later time.

The feasibility of the present QMD simulations has been made possible by the advances in both DFT methodology and computational capabilities that have occurred in the past two decades. This includes the development of robust all-electron and pseudopotential methods and the development of a parameter-free generalized gradient approximation (GGA ${ }^{40}$ for 
the exchange and correlation terms in DFT. This has resulted in a number of first-principles calculations of the ground-state properties of the light actinides that are able to reproduce the correct observed structures and obtain equilibrium volumes ${ }^{41-43}$ and elastic constants ${ }^{44,45}$ in close agreement with experiment. These calculations have made use of accurate but computationally expensive all-electron techniques such as full-potential linear muffin-tin orbital (FP-LMTO), full-potential linearized augmented plane-wave (FLAPW), and linear combinations of Gaussian-type orbitals fitting-function (LCGTO-FF). These methods have been mainly limited to the study of structures of small size or high symmetry because of their high computational cost. In addition, the difficulties within these approaches of accurately calculating forces and stresses have limited their usefulness for QMD. Recently, plane-wave pseudopotential calculations ${ }^{46}$ using GGA, but without the relativistic spin-orbit coupling, produced structural properties of complex low-symmetry compounds containing lanthanides and actinides, including uranium, with an accuracy comparable to that seen using DFT in materials containing lighter elements of the periodic table. Additional planewave pseudopotential DFT calculations ${ }^{47,48}$ of bulk uranium using GGA obtained the fully relaxed $\alpha-\mathrm{U}$ with structural parameters that differed from experiment by only a few percent and that compare favorably with previous all-electron calculations, putting uranium within reach of QMD.

\section{URANIUM PSEUDOPOTENTIAL AND LOW-TEMPERATURE PROPER- TIES}

To perform the QMD simulations discussed in this paper, we have constructed a planewave-based pseudopotential for uranium within DFT and the GGA for exchange and correlation of Perdew et al. ${ }^{40}$ by solving for the all-electron uranium atom $\mathrm{U}^{2.25+}$ in the reference state $6 s^{2} 6 p^{6} 6 d^{1} 5 f^{2.75}$. The energies of the core electrons were obtained by solving the scalar relativistic equations, while the valence electrons were treated non-relativistically. The pseu-

dopotential was modeled in the non-local norm-conserving Troullier-Martins form, ${ }^{49}$ with a total of 14 valence electrons generated using a plane-wave cutoff of $100 \mathrm{Ry}$. There is one projector for each component of angular momentum. The cutoff radii for the pseudized orbitals are $0.95,1.06,1.32$, and $0.95 \AA$ for the $6 s, 6 p, 6 d$, and $5 f$ valence orbitals, respectively. The $d$ component was chosen to be local. To improve the efficiency of our simulations, we 
transformed the pseudopotential into the Kleinman-Bylander form. ${ }^{50}$

The accuracy and transferability of our pseudopotential were tested by performing DFT calculations of the zero-temperature total energies of several different solid structures including bcc, fcc, hcp, body-centered tetragonal (bct), and $\alpha$-U. As noted above, $\alpha$-U is the stable phase of uranium at low temperatures up to pressures of $100 \mathrm{GPa}$, with a facecentered-orthorhombic $(\mathrm{Cmcm})$ structure that can be described with two atoms per unit cell with the basis vectors

$$
\begin{aligned}
\overrightarrow{\mathbf{B}}_{\mathbf{1}} & =y b \widehat{\mathbf{y}}+\frac{1}{4} c \widehat{\mathbf{z}} \\
\overrightarrow{\mathbf{B}}_{\mathbf{2}} & =-y b \widehat{\mathbf{y}}-\frac{1}{4} c \widehat{\mathbf{z}},
\end{aligned}
$$

where $b$ and $c$ are orthorhombic lattice parameters and $y$ is an internal parameter. The irreducible Brillouin zone was sampled using 44, 110, 150, 99, and 168 Monkhorst-Pack ${ }^{51}$ special $k$-points for the bcc, fcc, hcp, bct, and the $\alpha$-U structures, respectively. To improve convergence of the $k$-point integration, a Gaussian smearing of 0.02 Ry was used. Shown in Fig. 1 are the relative total energies for each of these structures computed at several different atomic volumes. At each volume the $c / a$ ratio in the bct structure was relaxed. The hcp structure was taken to be "ideal" with a fixed $c / a$ ratio of $\sqrt{8 / 3}$. The cell parameters $b / a$ and $c / a$ and the internal parameter $y$ of the $\alpha$-U were fully relaxed at each volume. We correctly obtained $\alpha$-U as the lowest-energy structure with an equilibrium atomic volume of $20.72 \AA^{3}$, in close agreement with the experimental value ${ }^{52}$ of $20.770 \AA^{3}$. Our result differs from previous calculations using different pseudopotentials constructed within the GGA, which obtained equilibrium volumes of $19.92 \AA^{3}$ with spin-orbit coupling included ${ }^{47}$ and $20.15 \AA^{3}$ without spin-orbit coupling. ${ }^{48}$ A Murnaghan fit ${ }^{53}$ to our total energy as a function of volume for $\alpha$-U yielded a bulk modulus of $133.5 \mathrm{GPa}$, which is larger than one reported experimental value ${ }^{52}$ of $104(2) \mathrm{GPa}$, but agrees closely with the DAC result of Yoo et al. ${ }^{33}$ of $135.5 \mathrm{GPa}$. Our pseudopotential results are also in close agreement with all-electron GGA FP-LMTO calculations ${ }^{44}$ on a fully relaxed $\alpha$-U structure, which gave an equilibrium volume of $20.67 \AA^{3}$ and a bulk modulus of $133.0 \mathrm{GPa}$. These latter calculations were fully relativistic and included spin-orbit coupling in a first-order variational treatment. Table I lists our calculated ground-state properties of $\alpha$-U compared with the FP-LMTO calculations and experimental data. Our calculated lattice parameters differ by at most $2 \%$ in comparison with experimental values and about $1 \%$ in comparison with the FP-LMTO 


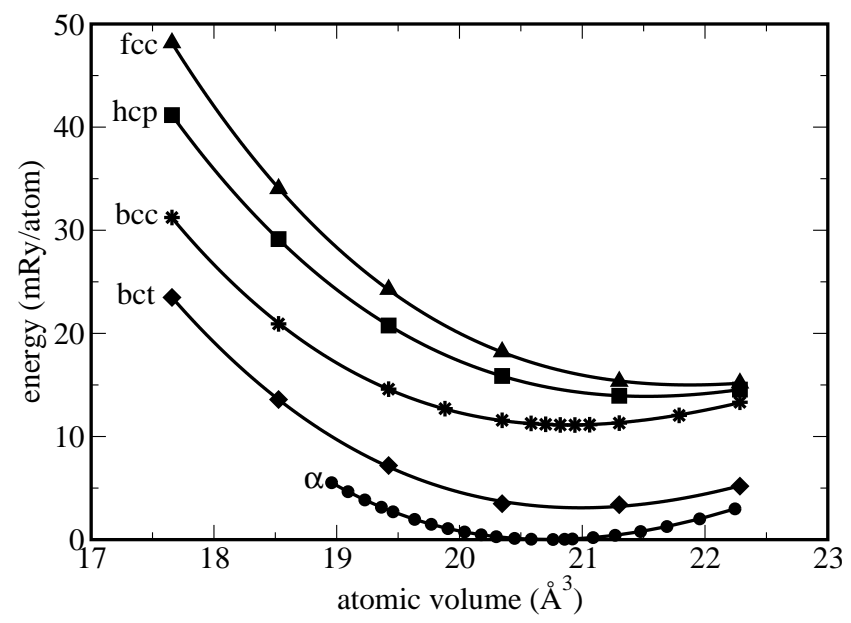

FIG. 1: Energies of different hypothetical phases of uranium in $\mathrm{mRy} /$ atom as a function of atomic volume $\left(\AA^{3}\right)$ relative to the observed $\alpha$-U phase at its equilibrium volume. Included are the relative energies of the body-centered tetragonal (bct), body-centered cubic (bcc), hexagonal-closed pack (hcp), and the face-centered cubic (fcc) structures.

results.

Up to $100 \mathrm{GPa}$, uranium has not been found to exist in the fcc, hcp, or bct structures, whose energies are plotted in Fig. 1, although FP-LMTO calculations ${ }^{16}$ predict that these structures will have lower energies than $\alpha$-U at large enough densities. As a further check of both the validity of our pseudopotential in the $100 \mathrm{GPa}$ pressure range and the impact of neglecting spin-orbit coupling, we have compared our calculated structural energies with the FP-LMTO results. ${ }^{16,44}$ Figure 2 shows that our pseudopotential is able to reproduce the correct energetic ordering of all five structures considered in comparison with FP-LMTO. This demonstrates that our plane-wave pseudopotential method is able to resolve the small energy differences between the high- and low-symmetry phases of uranium, even without including spin-orbit coupling. This is important for QMD simulations, where the local atomic environment can vary with time throughout the simulation cell.

Another useful validation test of our pseudopotential is to calculate structural energies along the continuous Bain path ${ }^{54}$ linking bcc and fcc at constant volume. Along this path, the bcc structure has one of its cubic directions labeled $c$ rather than $a$ to create a bct structure with variable $c / a$. When $c / a$ equals one the structure is bcc and when it equals $\sqrt{2}$ the structure is fcc. We performed Bain-path pseudopotential calculations at an atomic volume of $20.75 \AA^{3}$ with $c / a$ ranging between 0.75 and 1.8. As shown in Fig. 3, our results 
TABLE I: Ground-state properties of $\alpha$-U calculated from an all-electron FP-LTMO approach (Ref. 44) and from the present plane-wave pseudopotential approach, as compared with experiment. The equilibrium volume and the lattice constants $a, b$ and $c$ are in units of $\stackrel{\AA}{A}$, while the bulk modulus is in GPa. The experimental results (Ref. 52) were measured at room temperature.

\begin{tabular}{cccc}
\hline \hline & FP-LMTO & pseudopotential & Experiment \\
\hline volume & 20.67 & 20.72 & 20.770 \\
$a$ & 2.845 & 2.86 & 2.8553 \\
$b$ & 5.816 & 5.77 & 5.8702 \\
$c$ & 4.996 & 5.01 & 4.9568 \\
$y$ & 0.1025 & 0.104 & 0.102 \\
$B$ & 133.0 & 133.5 & $104(2), 135.5^{a}$ \\
$\mathrm{~B}^{\prime}$ & 5.4 & 5.0 & $6.2,3.8^{a}$ \\
\hline \hline
\end{tabular}

${ }^{a}$ Ref. 33

reproduce the qualitative behavior, and for $c / a$ between 0.75 and 1.08 the quantitative behavior, that is seen in corresponding FP-LMTO calculations, ${ }^{16}$ including a minimum in the total energy at the same $c / a$ ratio near 0.82 . The bcc structure is found to be mechanically unstable to tetragonal distortions in both calculations, with a negative curvature in the Bain path at $c / a=1$ and a negative $C^{\prime}$ elastic constant directly calculated. Thus in our QMD simulations we indeed expect bcc to be mechanically unstable at low temperature.

\section{QMD RESULTS AND DISCUSSION}

In QMD the ions move according to Newton's classical equations of motion in which the forces acting on the ions are computed "on-the-fly" by solving the DFT quantum mechanical equations for the electrons at each discrete time step. Newton's time-dependent equation was discretized using a Verlet leap-frog algorithm. ${ }^{55,56}$ We have used Born-Oppenheimer QMD in which the low lying single-particle electronic eigenstates are computed by solving the selfconsistent DFT Kohn-Sham equations ${ }^{2}$ within the framework of Mermin's finite temperature density functional theory. ${ }^{57}$ To accomplish this we used a preconditioned conjugate gradient method $^{3,58,59}$ to fully relax the electronic wavefunctions at each time step. An efficient fast 


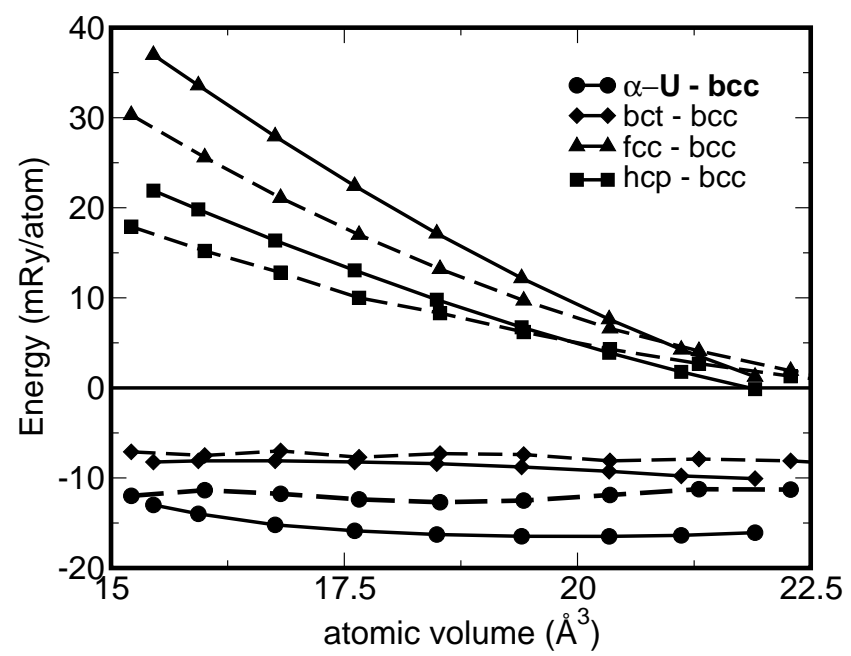

FIG. 2: Energy differences for uranium in the $\alpha$-U, body-centered tetragonal (bct), hexagonalclosed pack (hcp), and the face-centered cubic (fcc) structures relative to body-centered cubic (bcc), in units of mRy/atom as a function of volume per atom $\left(\AA^{3}\right)$. Solid lines are the energy differences computed using GGA FP-LMTO with spin-orbit coupling (Refs. 44 and 16). Dashed lines are our computed energy differences using a GGA pseudopotential without spin-orbit coupling.

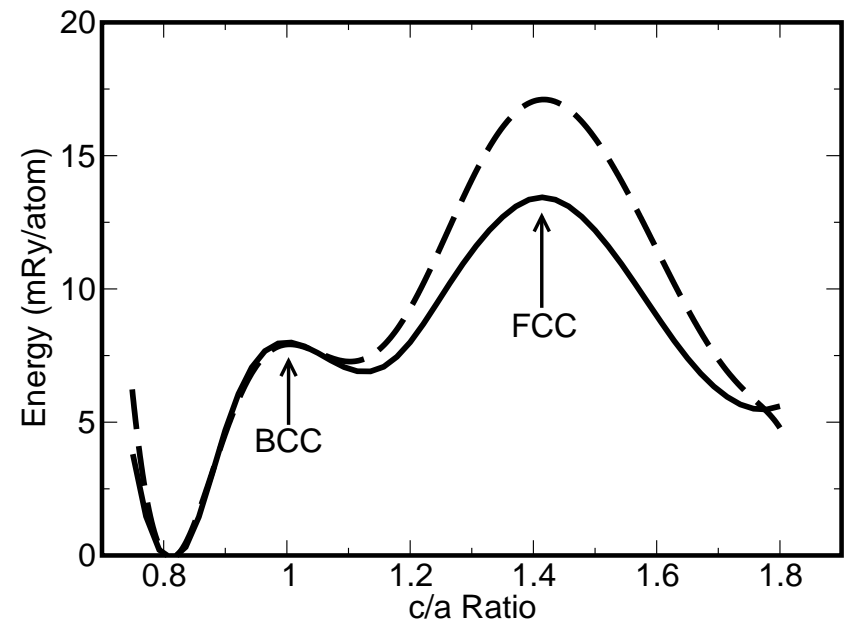

FIG. 3: The calculated constant-volume Bain path for uranium at an atomic volume $20.75 \AA^{3}$, which is close to the experimental equilibrium atomic volume at ambient conditions $20.770 \AA^{3}$ (Ref. 52). The solid line was computed using FP-LMTO (Ref. 16), while the dashed line was calculated using our present pseudopotential. Here $c / a=1$ and $c / a=\sqrt{2}$ correspond to the bcc and fcc structures, respectively. 
Fourier transform algorithm was used for the conversion of the wave functions between real and reciprocal spaces. ${ }^{59}$ The electronic eigenstates were thermally occupied using the FermiDirac distribution function at a temperature $T_{\text {electron }}$ equivalent to the ion temperature. The use of a pseudopotential along with a plane-wave basis allowed us to accurately calculate the forces acting on the ions. We have performed all of our QMD simulations in an NVT ensemble in which the volume is held constant within a fixed-shape simulation cell and the temperature is controlled using a Nose-Hoover thermostat. ${ }^{60}$

We have obtained full QMD results at nine chosen temperature-volume points using a cubic simulation cell containing 54 uranium atoms with periodic boundary conditions, a single $k$ point for Brillouin-zone averaging in reciprocal space, and a time-step of 1.2 fs. The chosen temperatures and corresponding calculated pressures are shown as circles and diamonds superimposed onto the experimental phase diagram of uranium in Fig. 4. The temperatures and volumes of the QMD simulations were chosen to span the observed solid $\alpha$-orthorhombic and $\gamma$-bcc phases and the liquid phase. Our small cubic simulation cell is readily commensurate with the high-temperature bcc and liquid phases, and we have observed these phases free of any external strains. This is not the case, however, for the low-temperature orthorhombic $\alpha$-U phase. As discussed below, we believe that we never actually achieved a relaxed $\alpha$-U structure in our QMD simulations nor the expected $\alpha$-U to bcc solid-solid phase transition. More likely, our low-temperature structure was bct or some simple distortion of bct, consistent with Figs. 2 and 3, and that this structure transformed continuously to bcc at high temperature. In contrast, clear evidence of melting from bcc to liquid was seen in our two highest temperature points, as also discussed below.

The starting ionic configuration for our lowest temperature simulation at $330 \mathrm{~K}$ was the bcc structure. Because bcc is mechanically unstable at these conditions, this structure immediately relaxed to a local energy minimum, which was undetermined but presumably bct-like. This metastable configuration was then thermalized for 0.5 ps before statistics were accumulated. The final configuration from this simulation was used as the starting configuration at $490 \mathrm{~K}$, which was again thermalized for 0.5 ps before statistics were accumulated. This procedure was repeated for each successively higher temperature simulation performed at the atomic volume $20.45 \AA^{3}$. In addition, to ensure that a good equilibrium was established for the two liquid states, we repeated the simulations for these states with different starting ionic configurations. These states were first brought to approximately 


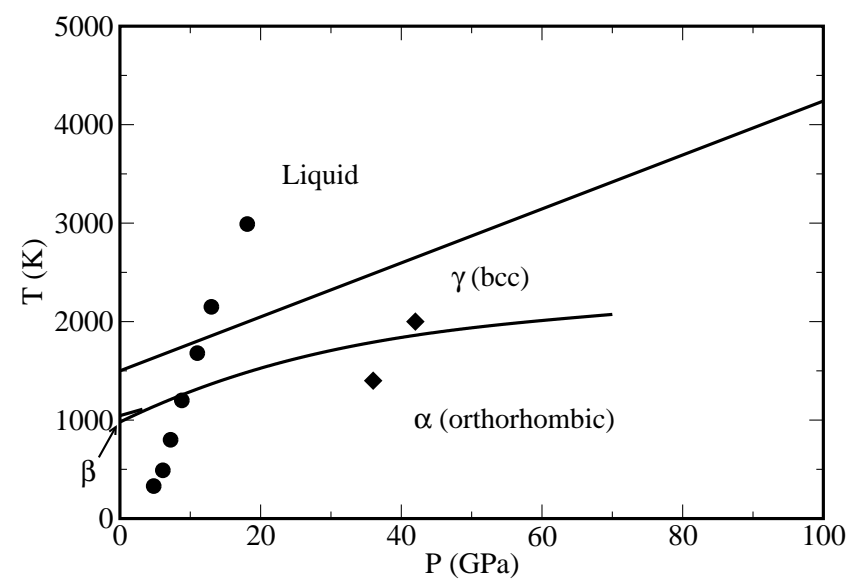

FIG. 4: Phase diagram of uranium to 100 GPa determined from in situ diamond-anvil-cell $\mathrm{x}$ ray/laser-heating experiments ${ }^{31,33}$ together with the present calculated EOS points. The circles (atomic volume $20.45 \AA^{3}$ ), and diamonds (atomic volume $17.53 \AA^{3}$ ) are positioned at the chosen temperatures and calculated pressures obtained using NVT-ensemble quantum molecular dynamics simulations. The statistical error bars in the pressures are smaller than the size of the symbols.

twice their respective final temperatures, 4000 and $6000 \mathrm{~K}$, and then thermalized at the higher temperatures for 0.5 ps before being slowly cooled down to 2150 and $2990 \mathrm{~K}$ and equilibrated to accumulate statistics. These liquid state results were independent, to within statistics, of the starting ionic configurations. The seven QMD data points shown as circles in Fig. 4 were simulated for times ranging from 1 ps (solid states) to 3-3.5 ps (liquid states). The two points shown as diamonds in Fig. 4 had a constant atomic volume of $17.53 \AA^{3}$ and were simulated for $3.7 \mathrm{ps}$, after starting each simulation in a bcc structure that was first thermalized for 0.5 ps.

In all of these QMD simulations, we expanded our electronic wavefunctions in a planewave basis with either an 80 Ry cutoff (solid states at $20.45 \AA^{3}$ ) or a 100 Ry cutoff (remaining states). We found that these cutoffs were sufficient to reproduce the calculated pressures to within our statistical error bars that were obtained using 100 or 120 Ry cutoffs, respectively. Table II lists the nine pressures and total energies that were so computed on the equation of state of uranium.

An extremely useful quantity in characterizing the theoretical high-pressure equation of state of a material is the Grüneisen parameter $\gamma_{\mathrm{G}}$ and its volume and temperature depen- 
TABLE II: Constant-temperature, constant-volume quantum molecular dynamics results for the equation of state of uranium computed at a range of chosen temperatures $\mathrm{T}$ and atomic volumes V. Included are the calculated pressures $\mathrm{P}$ and total energies E.

\begin{tabular}{rccc}
\hline \hline $\mathrm{T}(\mathrm{K})$ & $\mathrm{V}\left(\AA^{3} /\right.$ atom $)$ & $\mathrm{P}(\mathrm{GPa})$ & $\mathrm{E}(\mathrm{Ry} /$ atom $)$ \\
\hline 330 & 20.45 & 4.8 & $-102.573(1)$ \\
490 & 20.45 & 6.1 & $-102.570(1)$ \\
800 & 20.45 & 7.2 & $-102.562(1)$ \\
1200 & 20.45 & 8.8 & $-102.551(2)$ \\
1680 & 20.45 & 11.0 & $-102.542(2)$ \\
2150 & 20.45 & 13.0 & $-102.530(2)$ \\
2990 & 20.45 & 18.1 & $-102.554(2)$ \\
1400 & 17.53 & 36.0 & $-102.541(1)$ \\
2000 & 17.53 & 42.0 & $-102.533(1)$ \\
\hline \hline
\end{tabular}

dence. The Grüneisen parameter is defined as

$$
\gamma_{\mathrm{G}}=V\left(\frac{\partial P}{\partial E}\right)_{V}
$$

which via the chain rule can be written in terms of the temperature derivatives of the pressure, $\partial P / \partial T$, and the total energy, $\partial E / \partial T$, at constant volume. From the QMD data presented in Fig. 4 and Table II, it can be seen that $P$ and $E$ vary smoothly and almost linearly with temperature at constant volume, even across phase boundaries, indicating a near constant value of $\gamma_{\mathrm{G}}$ at those conditions. Assuming an exact linear temperature dependence at the atomic volume $20.45 \AA^{3}$, we calculate $\gamma_{\mathrm{G}}$ to be 1.7 for uranium. For comparison, the experimental value of $\gamma_{\mathrm{G}}$ at ambient conditions in the $\alpha$-U phase is 2.16, a value corresponding to an atomic volume of $20.77 \AA^{3}$ and obtained from the thermodynamic relation

$$
\gamma_{\mathrm{G}}=\frac{V \beta B_{S}}{C_{P}}
$$

through measured values of the thermal expansion coefficient $\beta$, adiabatic bulk modulus $B_{S}$ and constant-pressure specific heat $C_{P} \cdot{ }^{61}$ Similarly in the liquid at $T=1810 \mathrm{~K}$ and $P=$ $0.12 \mathrm{GPa}$, the experimental value ${ }^{62}$ determined for $\gamma_{\mathrm{G}}$ is 2.29 , corresponding to an atomic 
volume of $25.06 \AA^{3}$. If one assumes a local linear variation of $\gamma_{\mathrm{G}}$ with volume,

$$
\gamma_{\mathrm{G}}(V)=\gamma_{\mathrm{G}}^{0} V / V_{0}
$$

then this latter value reduces to 1.87 at an atomic volume of $20.45 \AA^{3}$, which is indeed close to our calculated result of 1.7 . In this regard, Yoo, et $a l^{31}$ found that the melting curve of uranium below 50 GPa obeyed a Lindemann scaling law based on an ion-Güneisen parameter with a linear volume dependence as in Eq. (3).

As noted above, the total energies from Table II at the atomic volume $20.45 \AA^{3}$ depend nearly linearly on the temperature. From the slope of this line we calculated the specific heat at constant volume,

$$
C_{V}=\left(\frac{\partial E}{\partial T}\right)_{V},
$$

to be $130 \mathrm{~J} / \mathrm{kg} \cdot \mathrm{K}$. Using the experimental specific heats ratio $C_{P} / C_{V}=1.5$ determined at $T$ $=1810 \mathrm{~K}$ in the liquid ${ }^{62}$ we estimate $C_{P}$ to be $195 \mathrm{~J} / \mathrm{kg} \cdot \mathrm{K}$ in liquid uranium. This compares favorably with the experimental value for $C_{P}$ of $210 \mathrm{~J} / \mathrm{kg} \cdot \mathrm{K}$ in the liquid state obtained at the atomic volume $25.06 \AA^{3}$ from isobaric expansion measurements. ${ }^{63}$

In addition to thermodynamic properties, we have also obtained a large quantity of atomic and electronic structural information as well as liquid transport properties from our QMD simulations. We have calculated both radial and angular distribution functions at each of our nine temperature-volume points. In Fig. 5 we show the calculated radial distribution function $g(r)$ for uranium at the atomic volume $20.45 \AA^{3}$ and the four temperatures $330,1680,2150$, and $2990 \mathrm{~K}$. Corresponding results for the bond-angle distribution function $g_{3}(\theta)$ are shown in Fig. 6. Here $g_{3}(\theta)$ has been calculated with a cutoff radius of $3.9 \AA$, which corresponds to the first minimum in $g(r)$. At the constant volume of $20.45 \AA^{3} /$ atom, both $g(r)$ and $g_{3}(\theta)$ changed smoothly with increasing temperature between 330 and $2990 \mathrm{~K}$. In the solid, we did not see changes in either function with increasing temperature that would indicate a solidsolid structural phase transition, such as that expected in uranium from the orthorhombic $\alpha$ $\mathrm{U}$ phase to the bcc $\gamma$ phase. At $1680 \mathrm{~K}$, the radial and bond-angle distribution functions are very characteristic of the high-temperature bcc solid. ${ }^{64}$ In contrast at $330 \mathrm{~K}$, the distribution functions, while similar, are too broad and with additional shoulders to be those of a lowtemperature bcc or other crystalline structure. These observations are consistent with a scenario of a distorted bct-like structure at low temperature continuously evolving into bcc at high temperature. Finally, at our two highest temperatures, 2150 and $2990 \mathrm{~K}$, clear 


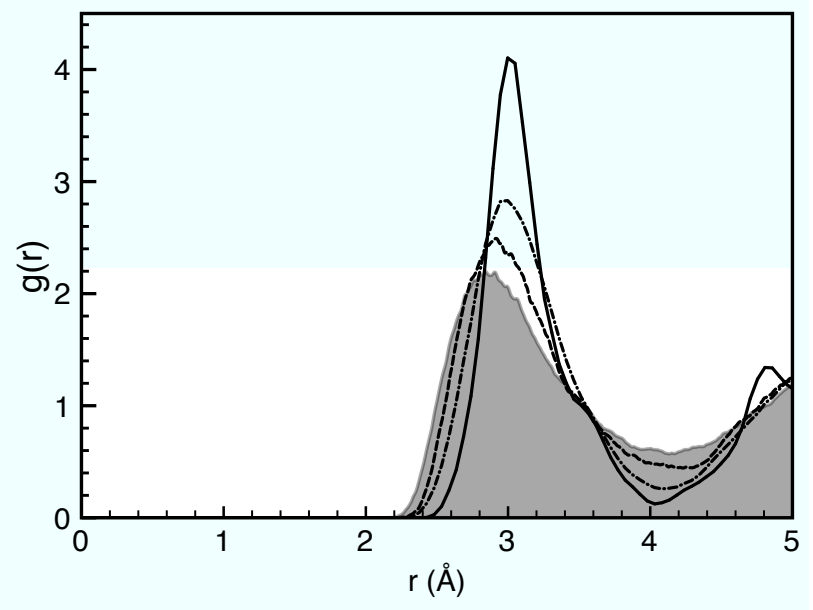

FIG. 5: Radial distribution function $g(r)$ for uranium calculated using QMD at an atomic volume of $20.45 \AA^{3}$ and the temperatures $330 \mathrm{~K}$ (solid line), $1680 \mathrm{~K}$ (dot-dashed line), $2150 \mathrm{~K}$ (dashed line), and $2990 \mathrm{~K}$ (thin solid line with shaded area).

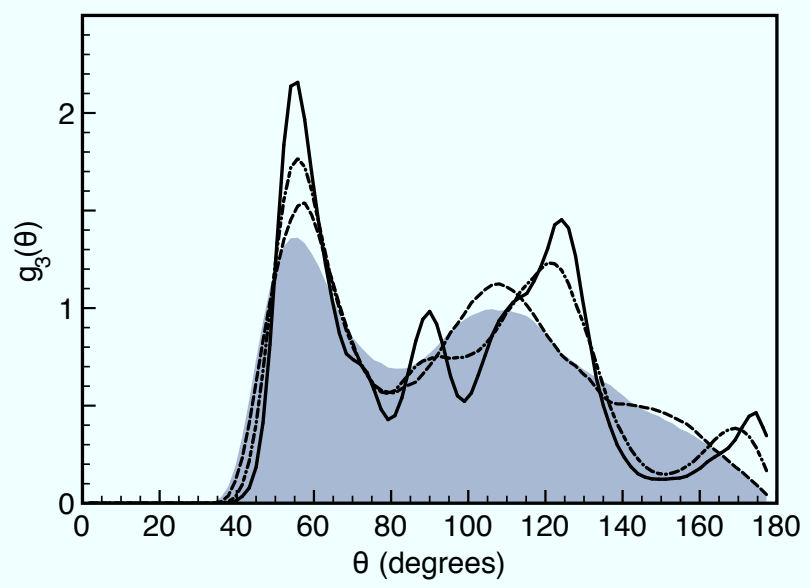

FIG. 6: Bond-angle distribution function $g_{3}(\theta)$ for uranium from QMD simulations at an atomic volume of $20.45 \AA^{3}$ and temperatures $330 \mathrm{~K}$ (solid line), $1680 \mathrm{~K}$ (dot-dashed line), $2150 \mathrm{~K}$ (dashed line), and $2990 \mathrm{~K}$ (thin solid line with shaded area).

evidence of melting and transition to a liquid state, $l$-U, is seen. In $g(r)$ this evidence comes from the first minimum near $3.9 \stackrel{\circ}{A}$, which is deep and near zero for the solid, but is considerably higher for the liquid. In $g_{3}(\theta)$, the small signature peak near $180^{\circ}$, which is characteristic of a cubic or tetragonal solid, is no longer present in the 2150- and 2990-K simulations. 


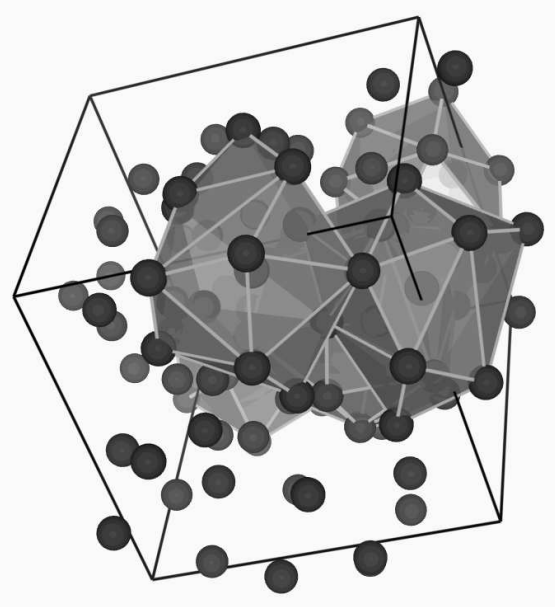

FIG. 7: Snapshot of the $l-\mathrm{U}$ structure at $T=2990 \mathrm{~K}$ showing a network of tetrahedra with a common vertex, which is a characteristic of short-range order (SRO) in the liquid.

At the same time, it is interesting to note that the liquid uranium ( $l$-U) states in our QMD results do show characteristics of short-range order (SRO). This can be seen in terms of the bond-angle distribution function $g_{3}(\theta)$ displayed in Fig. 6. The first peak in $g_{3}(\theta)$, at about $60^{\circ}$, corresponds to equilateral triangles, and the second peak, at around $109^{\circ}$, corresponds to the angle between tetrahedral edges that share a common face. Thus, the local structure with $\mathrm{SRO}$ in $l-\mathrm{U}$ is a collection of tetrahedra with a common vertex, as can be seen in the QMD snapshot presented in Fig. 7. The evolution of the SRO in the two $l$-U states we have simulated is also seen through the temperature dependence of $g_{3}(\theta)$. The amplitudes of the two peaks in $g_{3}(\theta)$ decrease with increasing temperature indicating a reduction in SRO as temperature increases. A more subtle feature is the shoulder around $150^{\circ}$ that appears in $g_{3}(\theta)$ at $2150 \mathrm{~K}$ but is absent at $2990 \mathrm{~K}$. The appearance of the shoulder suggests decreased stability and that one is near the freezing point for the liquid or even possibly in an undercooled metastable state. From Fig. 4 one sees that at $2150 \mathrm{~K}$ one is only a few hundred degrees above the experimental melt curve.

Additional confirmation of the liquid nature of our two highest temperature $l$ - $U$ states can be seen from the calculated mean square displacement (MSD) for the ions at long time, as is shown in Fig. 8. A positive slope to the asymptote of the MSD curve corresponds to diffusion of the ions away from their initial positions. From the long-time MSD slope one 


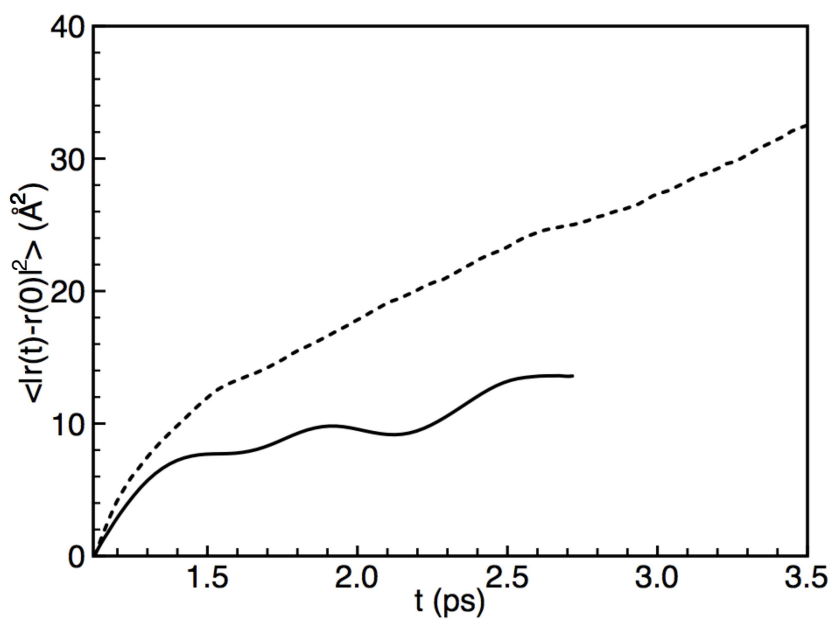

FIG. 8: Mean square displacement (MSD) for the $l-U$ states versus time as calculated from the present QMD simulations at an atomic volume of $20.45 \AA^{3}$ and temperatures $2150 \mathrm{~K}$ (solid curve) and $2990 \mathrm{~K}$ (dashed curve).

can calculate the liquid diffusion coefficient $D$ through the Einstein relation

$$
6 D=\lim _{t \rightarrow \infty} \frac{d}{d t}\left\langle\left|r_{i}(t)-r_{i}(0)\right|^{2}\right\rangle
$$

As expected, a higher diffusion coefficient $\left(1.71 \times 10^{-4} \mathrm{~cm}^{2} \mathrm{~s}^{-1}\right)$ is calculated for $l$ - $\mathrm{U}$ at $T=$ $2990 \mathrm{~K}$ than that at $T=2150 \mathrm{~K}$, where $D=0.92 \times 10^{-4} \mathrm{~cm}^{2} \mathrm{~s}^{-1}$.

Finally, the calculated total electronic densities of states from our $l-U$ QMD simulations are shown in Fig. 9. The corresponding results for the solid $\alpha$-U and bec structures at $T$ $=0$ are also displayed for comparison. Overall, the density of states (DOS) does not show any dramatic changes with increasing temperature or phase change from solid phases to the liquid states, except that the temperature smooths the sharp features in the DOS and slightly broadens the band widths in the solid phases, with the strongest effects on the band of localized $6 p$ states around $18 \mathrm{eV}$ below the Fermi level.

\section{CONCLUSIONS}

To summarize, we have carried out 54-atom constant-volume, constant-temperature QMD simulations of uranium at two different volumes and a range of temperatures covering the observed $\alpha$-U, bcc, and liquid phases up to $2000 \mathrm{~K}$ and $42 \mathrm{GPa}$. To our knowledge these are the first reported QMD simulations of an actinide element. We believe that the present 


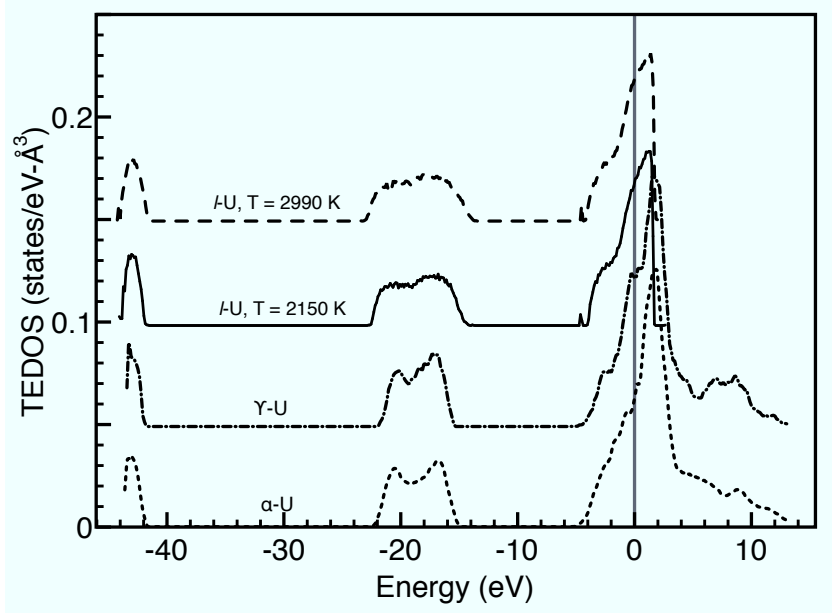

FIG. 9: Total electronic density of states (DOS) for uranium calculated from the present QMD simulations at an atomic volume of $20.45 \AA^{3}$ and temperatures $2150 \mathrm{~K}$ (solid line) and $2990 \mathrm{~K}$ (dashed line). The DOS for solid $\alpha$-U (dotted line) and bcc (dot-dashed) structures at $T=0$ are shown for comparison. The abrupt drop in the QMD DOS at $2 \mathrm{eV}$ is an artifact of using a limit number of unoccupied states. There are three main features in the each DOS: the low lying $6 s$ states around $-42 \mathrm{eV}$, the $6 p$ states around $-18 \mathrm{eV}$, and the higher energy $7 s, 6 d$, and $5 f$ valence states near the Fermi level.

plane-wave based pseudopotential approach within GGA of DFT gives an accurate description of uranium, and the specific pseudopotential we have developed for this work has been validated to $100 \mathrm{GPa}$. We have presented U simulation results for the equation of state, the Grüneisen parameter and specific heat, the radial and bond-angle distribution functions, the liquid diffusion constant, and the electronic density of states. From the bond-angle distribution for the liquid uranium states, we have found that the local atomic structure displays short range order. Among other things, these results should aid in the development of accurate multi-ion MGPT interatomic potentials ${ }^{39}$ that are valid at high temperatures and pressures, and can be used for in depth studies of additional structural, thermodynamic, defect and mechanical properties of uranium.

The primary limitation in our QMD simulations has been the necessary use of a small, fixed-shape (cubic) simulation cell. In the low and intermediate- temperature solid, this places external strains upon the system when the orthorhombic $\alpha$-U structure is the energetically preferred phase, although this is not the case at high temperature when uranium is 
in the bcc or liquid phases. The future development of constant-pressure, variable-cell QMD simulations may rectify the former difficulty, but this remains very challenging. In principle,

such simulations could accommodate the $\alpha$-U phase and its large thermal anisotropy, ${ }^{36}$ and permit the direct observation of the $\alpha$-U to bcc phase transition.

\section{ACKNOWLEDGMENTS}

This work was performed under the auspices of the U. S. Department of Energy by Lawrence Livermore National Laboratory in part under Contract W-7405-Eng-48 and in part under Contract DE-AC52-07NA27344. Our calculations were performed on the ASC Q machine at Los Alamos National Laboratory and on the ASC White and Atlas machines at Lawrence Livermore National Laboratory.

1 P. Hohenberg and W. Kohn, Phys. Rev. 136, B864 (1964).

2 W. Kohn and L. J. Sham, Phys. Rev. 140, A1133 (1965).

3 M. C. Payne, M. P. Teter, D. C. Allan, T. A. Arias, and J. D. Joannopoulos, Rev. Mod. Phys. 64, 1045 (1992).

4 D. Marx and J. Hütter, in 2000 Modern Methods and Algorithms of Quantum Chemistry, edited by J. Grotendorst (Forschungzentrum: Jülich NIC Series vol 1), pp. 301-449.

5 G. Kresse and J. Furthmüller, Comput. Mater. Sci. 6 15, (1996).

6 M. Parrinello, Solid State Commun. 102, 107 (1997).

7 G. A. de Wijs, G. Kresse, and M. J. Gillan, Phys. Rev. B 57, 8223 (1998).

8 S. Bernard and J. B. Maillet, Phys. Rev. B 66, 12103 (2002).

9 D. Alfe, M. J. Gillan, and G. D. Price, Nature 40, 462 (1999).

10 A. Laio, S. Bernard, G. L. Chiarotti, S. Scandolo, and E. Tosatti, Science 287, 1027 (2000).

11 A. B. Belonoshko, S. I. Simak, A. E. Kochetov, B. Johansson, L. Burakovsky, and D. L. Preston, Phys. Rev. Lett. 92, 195701 (2004).

12 C. Cazorla and M. J. Gillan, J. Chem. Phys. 126, 194502 (2007).

13 S. Taioli, C. Cazorla, M. J. Gillan, and D. Alfe, Phys. Rev. B 75, 214103 (2007).

14 J. J. Katz, G. T. Seaborg, and L. R. Morss, in The Chemistry of the Actinide Elements, 2nd 
ed., edited by J. J. Katz, L. R. Morss, and G. T. Seaborg (Chapman and Hall, New York, 1986), pp. 1121-1195.

15 M. S. S. Brooks, B. Johansson, and H. L. Skriver, in Handbook on the Physics and Chemistry of the Actinides (Ref. 1), Vol. 1, Chap. 3, pp. 227-269.

16 P. Söderlind, Adv. Phys. 47, 959 (1998).

17 H. G. Smith, N. Wakabayashi, W. P. Crummett, R. M. Nicklow, G. H. Lander, and E. S. Fisher, Phys. Rev. Lett. 44, 1612 (1980).

18 G. H. Lander, E. S. Fisher, and S. D. Bader, Adv. Phys. 43, 1 (1994).

19 E. J. Nelson, P. G. Allen, K. J. M. Blobaum, M. A. Wall, and C. H. Booth, Phys. Rev. B 71, $184113(2005)$.

20 R. Pynn, W. Press, S. M. Shapiro, and S. A. Werner, Phys. Rev. B 13, 295 (1976).

21 D. Gibbs, K. M. Mohanty, and J. Bohr, Phys. Rev. B 37, 562 (1988).

22 L. Fast, O. Eriksson, B. Johansson, J. M. Wills, G. Straub, H. Roeder, and L. Nordström, Phys. Rev. Lett. 81, 2978 (1998).

23 N. Stojić, J. W. Davenport, M. Komelj, and J. Glimm, Phys. Rev. B 68, 094407 (2003).

24 A. Goldoni, A. Baraldi, G. Comelli, S. Lizzit, and G. Paolucci, Phys. Rev. Lett. 82, 3156 (1999).

25 Y. G. Hao, O. Eriksson, G. W. Fernando, and B. R. Cooper, Phys. Rev. B 47, 6680 (1993).

26 M. E. Manley, M. Yethiraj, H. Sinn, H. M. Volz, A. Alatas, J. C. Lashley, W. L. Hults, G. H. Lander, and J. L. Smith, Phys. Rev. Lett. 96, 125501 (2006).

27 G. M. Schmiedeshoff, D. Dulguerova, J. Quan, S. Touton, C. H. Mielke, A. D. Christianson, A. H. Lacerda, E. Palm, S. T. Hannahs, T. Murphy, E. C. Gay, C. C. McPheeters, D. J. Thoma, W. L. Hults, J. C. Cooley, A. M. Kelly, R. J. Hanrahan, and J. L. Smith, Philos. Mag. 84, 2001 (2004).

28 E. S. Fisher, J. Alloys Compd. 213/214, 254 (1994).

29 J. Ganguly and G. C. Kennedy, J. Phys. Chem. Solids 34, 2272 (1973).

30 J. Akella, G. S. Smith, R. Grover, Y. Yu, and S. Martin, High Pressure Research 2, 295 (1990).

31 C. -S. Yoo, J. Akella, and J. A. Moriarty, Phys. Rev. B 48, 15529 (1993).

32 J. Akella, S. Weir, J. M. Wills, and P. Söderlind, J. Phys.: Condens. Matter 9, L549 (1997).

33 C. -S. Yoo, H. Cynn, and P. Söderlind, Phys. Rev. B 57, 10359 (1998).

34 R. W. G. Wyckoff, Crystal Structures (Wiley, New York, 1963), p. 16.

35 J. Donohue, The Structure of the Elements (Wiley, New York, 1974), Chap. 5. 
36 A. C. Lawson, C. E. Olsen, J. W. Richardson, Jr., M. H. Mueller, and G. H. Lander, Acta Crystallogr., Sect. B: Struct. Sci. 44, 89 (1988).

37 D. A. Young, Phase Diagrams of the Elements (University of California, Berkeley, 1991), p. 222.

38 J. A. Moriarty, Phys. Rev. B 42, 1609 (1990).

39 J. A. Moriarty, L. X. Benedict, J. N. Glosli, R. Q. Hood, D. A. Orlikowski, M. V. Patel, P. Söderlind, F. H. Streitz, M. Tang, and L. H. Yang, J. Mater. Res. 21, 563 (2006).

40 J. P. Perdew and Y. Wang, Phys. Rev. B 45, 13244 (1992); J. P. Perdew, K. Burke, and M. Ernzerhof, Phys. Rev. Lett. 77, 3865 (1996).

41 M. D. Jones, J. C. Boettger, R. C. Albers, and D. J. Singh, Phys. Rev. B 61, 4644 (2000).

42 P. Söderlind, O. Eriksson, B. Johansson, and J. M. Wills, Phys. Rev. B 50, 7291 (1994).

43 M. Pénicaud, J. Phys.: Condens. Matter 12, 5819 (2000).

44 P. Söderlind, Phys. Rev. B 66, 085113 (2002).

45 P. Söderlind, A. Landa, B. Sadigh, L. Vitos, and A. Ruban, Phys. Rev. B 70, 144103 (2004).

46 C. J. Pickard, B. Winkler, R. K. Chen, M. C. Payne, M. H. Lee, J. S. Lin, J. A. White, V. Milman, and D. Vanderbilt, Phys. Rev. Lett. 85, 5122 (2000).

47 N. Richard, S. Bernard, F. Jollet, and M. Torrent, Phys. Rev. B 66, 235112 (2002).

48 M. Freyss, T. Petit, and J. P. Crocombette, J. Nucl. Mater. 347, 44 (2005).

49 N. Troullier and J. L. Martins, Phys. Rev. B 43, 1993 (1991).

50 L. Kleinman and D. M. Bylander, Phys. Rev. Lett. 48, 1425 (1982).

51 H. J. Monkhorst and J. D. Pack, Phys. Rev. B 13, 5188 (1976); J. D. Pack and H. J. Monkhorst, Phys. Rev. B 16, 1748 (1977).

52 T. Le Bihan, S. Heathman, M. Idiri, G. H. Lander, J. M. Wills, A. C. Lawson, and A. Lindbaum, Phys. Rev. B 67, 134102 (2003).

53 F. D. Murnaghan, Proc. Natl. Acad. Sci. USA 30, 244 (1994).

54 E. C. Bain, Trans. Am. Inst. Min. Metall. Eng. 70, 25 (1924).

55 L. Verlet, Phys. Rev. 159, 98 (1967).

56 R. W. Hockney and J. W. Eastwood, Computer Simulation Using Particles (McGraw-Hill, New York, 1981).

57 N. D. Mermin, Phys. Rev. 137, A1441 (1965).

58 L. H. Yang, in Industrial Strength Parallel Computing, edited by A. Koniges (Morgan Kaufmann Publishers, San Francisco, 2000), p. 297. 
59 L. H. Yang, R. Q. Hood, J. E. Pask, and J. E. Klepeis, J. Computer Aided Mater. Des. 14, 337 (2007).

60 S. Nose, J. Chem. Phys. 81, 511 (1984); S. Nose, Mol. Phys. 52, 255 (1984); W. G. Hoover, Phys. Rev. A 31, 1695 (1985).

61 D. Gray, Coord. Ed., em American Institute of Physics Handbook, 3rd ed. (McGraw-Hill, San Francisco, 1972); G. Simmons and W. Wang, Eds., Single Crystal Elastic Constants and Calculated Aggregate Properties: A Handbook, 2nd ed. (MIT, Cambridge, 1971).

62 M. Boivineau, L. Arlès, J. M. Vermeulen, and Th. Thévenin, Physica B 190, 31 (1993).

63 R. N. R. Mulford and R. I. Sheldon, J. Nucl. Mater. 154, 268 (1988); R. I. Sheldon and R. N. R. Mulford, J. Nucl. Mater. 185, 297 (1991).

64 J. A. Moriarty, Phys. Rev. B 49, 12431 (1994). 